\title{
Early versus Delayed Fixed Dose Combination Abacavir/Lamivudine/Zidovudine in Patients with HIV and Tuberculosis in Tanzania
}

\author{
Humphrey J. Shao,,2 John A. Crump,, ${ }^{1,3,4}$ Habib O. Ramadhani, ${ }^{1,2}$ Leonard O. Uiso, \\ Sendui Ole-Nguyaine, ${ }^{1,2}$ Andrew M. Moon, ${ }^{3}$ Rehema A. Kiwera, ${ }^{6}$ Christopher W. Woods, ${ }^{3,4}$ \\ John F. Shao,, John A. Bartlett, ${ }^{1,2,3,4}$ and Nathan M. Thielman ${ }^{3,4}$
}

\begin{abstract}
Fixed dose combination abacavir/lamivudine/zidovudine (ABC/3TC/ZDV) among HIV-1 and tuberculosis (TB)-coinfected patients was evaluated and outcomes between early vs. delayed initiation were compared. In a randomized, pilot study conducted in the Kilimanjaro Region of Tanzania, HIV-infected inpatients with smearpositive TB and total lymphocyte count $<1200 / \mathrm{mm}^{3}$ were randomized to initiate ABC/3TC/ZDV either 2 (early) or 8 (delayed) weeks after commencing antituberculosis therapy and were followed for 104 weeks. Of 94 patients screened, 70 enrolled $\left(41 \%\right.$ female, median CD4 count 103 cells $/ \mathrm{mm}^{3}$ ), and 33 in each group completed 104 weeks. Two deaths and 12 serious adverse events (SAEs) were observed in the early arm vs. one death, one clinical failure, and seven SAEs in the delayed $\operatorname{arm}(p=0.6012$ for time to first grade $3 / 4$ event, SAE, or death). CD4 cell increases were +331 and +328 cells $/ \mathrm{mm}^{3}$, respectively. TB-immune reconstitution inflammatory syndromes (TB-IRIS) were not observed in any subject. Using intent-to-treat (ITT), missing $=$ failure analyses, $74 \%$ $(26 / 35)$ vs. $89 \%(31 / 35)$ randomized to early vs. delayed therapy had HIV RNA levels $<400$ copies/ml at 104 weeks $(p=0.2182)$ and $66 \%(23 / 35)$ vs. $74 \%(26 / 35)$, respectively, had HIV RNA levels $<50$ copies $/ \mathrm{ml}(p=$ 0.6026). In an analysis in which switches from $\mathrm{ABC} / 3 \mathrm{TC} / \mathrm{ZDV}=$ failure, those receiving early therapy were less likely to be suppressed to $<400$ copies $/ \mathrm{ml}[60 \%(21 / 35)$ vs. $86 \%(30 / 35), p=0.030]$. TB-IRIS was not observed among the 70 coinfected subjects beginning antiretroviral treatment. ABC/3TC/ZDV was well tolerated and resulted in steady immunologic improvement. Rates of virologic suppression were similar between early and delayed treatment strategies with triple nucleoside regimens when substitutions were allowed.
\end{abstract}

\section{Introduction}

$\mathbf{T}$ He Clinical MANAGEMENT OF COINFECTION with HIV and tuberculosis (TB) presents a number of challenges, particularly in Africa, where $85 \%$ of incident coinfections occur and where TB is the leading cause of HIV-associated mortality. ${ }^{1-6}$ Many questions surround the optimal clinical management of HIV and TB coinfection; these include timing of initiation of antiretroviral therapy (ART), management strategies for overlapping toxicities of antituberculous and antiretroviral medications, and drug interactions between rifampicin-based antituberculous regimens with nonnucleo- side reverse transcriptase inhibitors (NNRTIs) and protease inhibitors (PIs).

An important complication of initiating ART in coinfected patients is the paradoxical worsening of signs and symptoms of TB secondary to immunologic reconstitution. Commonly manifest by fevers, increased or initial appearance of adenopathy, new or worsening pulmonary infiltrates, serositis, cutaneous lesions, and new or expanding central nervous system mass lesions, this syndrome has been reported to occur in $8-43 \%$ of patients receiving ART. ${ }^{7-14}$ The risk is greatest among those with lower CD4 cell counts, $7,10,14$ a population for which prompt initiation of ART may be

\footnotetext{
${ }^{1}$ Kilimanjaro Christian Medical Centre, Moshi, Tanzania.

${ }^{2}$ Kilimanjaro Christian Medical College, Tumaini University, Moshi, Tanzania.

${ }^{3}$ Duke University Medical Center, Durham, North Carolina 27710.

${ }^{4}$ Duke Global Health Institute, Duke University, Durham, North Carolina 27710.

${ }^{5}$ Kibong'oto National Tuberculosis Hospital, Sanya Juu, Tanzania.

${ }^{6}$ Kikundi cha Wanawake Kilimanjaro Kupambana na Ukimwi (KIWAKKUKI), Moshi, Tanzania.
} 
life-saving, ${ }^{15-18}$ and for those with shorter intervals between initiation of ART and antituberculosis therapy. ${ }^{12,14,19,20}$

Thus, in an individual patient, the risk of deferring therapy to avoid TB-associated immune reconstitution inflammatory syndrome (TB-IRIS) must be balanced against the risk of death, particularly for patients with $<200$ CD4 cells $/ \mathrm{mm}^{3} \cdot{ }^{21-23}$ To date, no published prospective randomized study has examined the optimal timing of ART following initiation of anti-TB therapy.

Of the currently available NNRTIs and PIs, efavirenz is recommended as the drug of choice for patients requiring concomitant anti-TB therapy. ${ }^{24-26}$ However, non-efavirenzbased treatment strategies are needed for selected patients for several reasons: (1) efavirenz is not recommended for use in pregnancy, ${ }^{27}$ (2) perhaps in part because of the widespread use of nevirapine for the prevention of mother-to-child transmission, reports of virologic failure associated with NNRTI resistance are increasing, ${ }^{28}$ (3) a small but considerable proportion of patients in resource-poor settings discontinues this medication because of side effects, ${ }^{29}$ and (4) efavirenz-based regimens are more costly (approximately twice that of nevirapine-based regimens), ${ }^{30}$ limiting their availability in resource-poor settings. ${ }^{31}$

Abacavir/lamivudine/zidovudine (ABC/3TC/ZDV) has been suggested as an alternate regimen for patients who cannot take efavirenz, ${ }^{24,25,30}$ but limited data describe its use among coinfected patients. In a small study of 34 subjects with HIV and TB and with CD4 cell counts $>350$ cells $/ \mathrm{ml}$ at baseline, HIV RNA levels were suppressed to $<400$ and $<50$ copies $/ \mathrm{ml}$ in $91 \%$ and $76 \%$ of subjects, respectively, at 24 weeks, and no cases of abacavir hypersensitivity reactions (HSR) or TB-IRIS were reported. ${ }^{32}$ We designed a pilot study, the Tuberculosis and HIV Immune Reconstitution Syndrome Trial (THIRST), to further assess the efficacy and safety of $\mathrm{ABC} / 3 \mathrm{TC} / \mathrm{ZDV}$ among coinfected patients and to compare outcomes, including the emergence of TB-IRIS, between subjects randomized to early vs. delayed ART, relative to the initiation of anti-TB therapy.

\section{Materials and Methods}

\section{Study subjects and design}

Eligible patients were HIV-1-infected adults aged 13 years and older who had received no previous ART (unless received for the prevention of mother-to-child transmission of HIV), had been admitted to one of two hospitals within 56 days of a diagnosis of pulmonary or extrapulmonary TB based on acid fast smear positivity, had received $<14$ days of anti-TB therapy, and had a total lymphocyte count $\leq 1200 / \mathrm{mm}^{3}$. The study was approved by the Institutional Review Board of Duke University Medical Center, the Kilimanjaro Christian Medical Centre (KCMC) Research Ethics Committee, and the Medical Research Coordinating Committee of the National Institute of Medical Research in Tanzania, and all subjects provided written informed consent. A protocol-mandated interim safety review of serious adverse events was completed after 10 patients from each group had received at least 8 weeks of therapy, and results were reported to each ethical review board and the Tanzanian Food and Drugs Authority. This study is registered with ClinicalTrials.gov, number NCT00851630.

Screening evaluations included a review of HIV and TB treatment history, documentation of HIV testing, complete blood cell count with differential, alanine aminotransferase (ALT), and serum creatinine. Prior to initiating the trial, study subject numbers were randomly assigned 1:1 to early vs. delayed treatment groups by a computer algorithm, and study team members not involved in subject recruitment or enrollment placed assignments into sealed opaque envelopes that were opened once participants met eligibility criteria. Eligible, consenting subjects were thus randomized with equal probability to initiate fixed dose combination abacavir $(300 \mathrm{mg}) /$ lamivudine $(150 \mathrm{mg})$ /zidovudine $(300 \mathrm{mg}$ ) administered orally twice daily, either 2 weeks (early group) or 8 weeks (delayed group) after starting anti-TB medications.

The planned duration of this study was 104 weeks from initiation of ART. Baseline evaluations included a medical history, clinical assessment, and laboratory tests (see below) including CD4 cell count. Plasma was archived for HIV RNA testing.

All study subjects remained as inpatients from the time of initiation of anti-TB therapy until completion of their eighth week of ART. If subjects developed suspected abacavir HSR, fixed dose combination ABC/3TC/ZDV was discontinued, and patients were given tenofovir $300 \mathrm{mg}$ by mouth once daily, lamivudine $150 \mathrm{mg}$ by mouth twice daily, and zidovudine $300 \mathrm{mg}$ by mouth twice daily. For study subjects suspected of zidovudine-related toxicities, fixed-dose combination $\mathrm{ABC} / 3 \mathrm{TC} / \mathrm{ZDV}$ was discontinued, and patients commenced abacavir $300 \mathrm{mg}$ twice daily, lamivudine $150 \mathrm{mg}$ twice daily, and stavudine $40 \mathrm{mg}$ twice daily (30 $\mathrm{mg}$ for those with weighing $<60 \mathrm{~kg}$ ).

\section{Clinical assessments}

Subjects were evaluated weekly for the first 8 weeks as inpatients following initiation of $\mathrm{ABC} / 3 \mathrm{TC} / \mathrm{ZDV}$ and monthly at KCMC thereafter. Subjects were assessed specifically for the development of TB-IRIS and abacavir HSR during each visit. TB-IRIS was defined by the protocol as (1) new persistent fevers (temperature $>101.5^{\circ} \mathrm{F}$ ) developing after the initiation of ART, not believed to be associated with ART, and without an identifiable source, (2) marked worsening or emergence of intrathoracic lymphadenopathy, pulmonary infiltrates, or pleural effusions on radiologic examination, or (3) worsening or emergence of lymphadenopathy on serial examinations or worsening of other TB lesions. Study personnel received specific training to assess for abacavir HSR. Trained workers from the community-based organization Kikundi cha Wanawake Kilimanjaro Kupambana na Ukimwi (KIWAKKUKI) conducted home visits to encourage adherence.

\section{Laboratory assessments}

CD4 cell counts, complete blood cell counts (CBCs), ALT, serum creatinine, glucose, and erythrocyte sedimentation rate (ESR) measurements were taken at weeks 12, 24, 36, 48, 60, 72, 84,96 , and 104, and plasma was archived from each of these visits. More intensive toxicity monitoring during the first 2 months of ART included CBCs, creatinine, glucose, ALT, and ESR at weeks 4 and 8. Toxicities were graded using NIAID Division of AIDS criteria. CD4 cell counts were performed using Coulter Manual CD4 Count Kits (Beckman Coulter, Inc., Fullerton, CA). Plasma was archived for HIV RNA testing and shipped in three batches to the Durham Veterans Affairs Medical Center in Durham, NC, for HIV-1 RNA 
quantification using Roche Amplicor HIV-1 Monitor Test version 1.5 (Roche Molecular Systems, Inc., Branchburg, NJ).

\section{Statistical analysis}

The primary objectives of this study were (1) to assess the feasibility and safety of a fixed-dose combination ABC/3TC/ ZDV in HIV-infected subjects with TB in a resource-limited setting and (2) to assess the impact of delayed vs. early initiation strategy for fixed-dose combination ABC/3TC/ZDV on the rate of TB-IRIS. Based on literature available at the time the study was designed, the anticipated incidence of TB-IRIS for patients initiating antiretroviral and antituberculosis medications was $30 \% .{ }^{11,12}$ Estimating that $30 \%$ and $5 \%$ of patients receiving early and delayed ART, respectively, would develop TB-IRIS, a sample size of 35 patients within each group (with allowance of up to seven patients to drop out of each group) was chosen to provide $80 \%$ power with alpha $=0.05$ (one-sided).

Planned analyses included comparing the proportion of subjects demonstrating TB-IRIS and other adverse events in early vs. delayed groups, and descriptions of clinical, immunologic, and virologic responses. Safety in each arm was assessed using Cox proportional hazards modeling of time to first grade $3 / 4$ event, serious adverse event, or death. The primary analysis of virologic responses to early vs. delayed strategies was intent to treat (ITT), missing = failure, in which protocol-defined substitutions were allowed. A secondary ITT analysis, missing $=$ failure, was performed in which switches from fixed dose combination $\mathrm{ABC} / 3 \mathrm{TC} / \mathrm{ZDV}$ were considered failures.

Differences in proportions of subjects with HIV RNA levels $<50$ and $<400$ copies $/ \mathrm{ml}$ between early vs. delayed groups were compared using Fisher's exact test. Statistical analyses were calculated using JMP 7.0 (SAS Institute Inc., Cary, NC).

\section{Results}

\section{Subject screening and baseline characteristics}

Enrollment began in June 2004 and was completed in September 2005. The last subject completed 104 weeks of study therapy in September 2007. The disposition of study subjects from recruitment through 104 weeks of follow-up is shown in Fig. 1. Baseline demographic and disease characteristics at study entry (summarized in Table 1) were comparable across treatment arms with the exception of weight.

\section{Subject disposition, safety, and TB-IRIS events}

Of the 35 subjects randomized to receive early $\mathrm{ABC} / 3 \mathrm{TC} / \mathrm{ZDV}$, two switched to abacavir, lamivudine, stavudine because of zidovudine-associated anemia, and three switched to tenofovir, lamivudine, zidovudine due to suspected abacavir HSR. Two subjects died, one at week 20 with a tension pneumothorax and underlying probable pneumoconiosis and the other at week 35 with cerebral malaria; neither death was judged by the investigators at the time to be related to TB-IRIS. Altogether, 12 serious adverse events were recorded among patients in the early arm (see Table 2).

Among the 35 subjects randomized to delayed therapy, one switched to tenofovir, lamivudine, zidovudine for suspected abacavir HSR, one died of complications thought to be related to disseminated Kaposi's sarcoma at week 4 , and another ex- perienced overt clinical antiretroviral failure at week 86 and was removed from the study. This subject was eventually documented to have a bloodstream infection with $M$. sherri$s i i .^{33} \mathrm{~A}$ total of seven serious adverse events were documented among patients in the delayed arm. Overall five subjects in the early group and one subject in the delayed group were switched from their initial antiretroviral regimen of $\mathrm{ABC} / 3 \mathrm{TC} /$ ZDV due to drug-related toxicity ( $p=0.3565)$.

Cox proportional hazards modeling of time to first event (grade 3 or 4 toxicity, serious adverse event or death) showed no difference between early vs. delayed treatment arms $(p=0.6012)$. No participants from either arm of the study had a protocol-defined TB-IRIS event throughout the 1753 patientmonths of follow-up, including inpatient monitoring for the first 8 weeks of ART for all subjects. At week 104, across both study arms, $60(86 \%)$ of subjects remained on ABC/3TC/ZDV and $66(94 \%)$ remained on study, receiving a protocol-defined regimen. Twenty-eight $(80 \%)$ vs. $32(91 \%)$ of subjects randomized to the early vs. delayed arms remained on $A B C /$ 3TC/ZDV at week $104(p=0.1719)$.

\section{Immunologic and virologic responses}

Figure 2 shows CD4 cell counts over time, by treatment group. The median increase in CD4 cell count from study entry to week 104 was 329 (IQR, 329-384) cells $/ \mathrm{mm}^{3}$. There was no difference in the CD4 cell increases between subjects randomized to the early treatment arm, 331 (IQR, 313376) cells $/ \mathrm{mm}^{3}$, or the delayed treatment arm, 328 (IQR, 281399) cells $/ \mathrm{mm}^{3}$.

Plasma HIV RNA levels could not be measured on the samples obtained at study entry and week 24 . Among samples from week 48, HIV RNA was detected in 34, not detected in 32 , and could not be performed in one sample. In ITT analyses, $32(91 \%)$ and $31(89 \%)$ of subjects randomized to early and delayed arms, respectively, had HIV RNA levels $<400$ copies $(p=1.0)$, and $24(69 \%)$ and $19(54 \%)$, respectively, had HIV RNA levels $<50$ copies $/ \mathrm{ml}(p=0.3261)$. Among all 70 subjects at week $48,63(90 \%)$ were suppressed to $<400$ copies $/ \mathrm{ml}$ and $43(61 \%)$ to $<50$ copies $/ \mathrm{ml}$.

The proportions of subjects with undetectable plasma HIV RNA levels at $<400$ copies $/ \mathrm{ml}$ and $<50$ copies $/ \mathrm{ml}$ after 104 weeks are shown in Fig. 3. Plasma HIV RNA levels were $<400$ copies $/ \mathrm{ml}$ in $26(74 \%)$ and $31(89 \%)$ of subjects randomized to early vs. delayed strategies, respectively ( $p=$ $0.2182)$, and $<50$ copies/ $\mathrm{ml}$ in $23(66 \%)$ vs. $26(74 \%)$, respectively ( $p=0.6026)$. Using intent to treat analyses, overall, 57 $(81 \%)$ and $49(70 \%)$ were suppressed to $<400$ copies $/ \mathrm{ml}$ and $<50$ copies $/ \mathrm{ml}$, respectively, at 104 weeks. In an analysis in which switches from fixed dose combination ABC/3TC/ZDV were considered failures, those receiving early therapy were less likely to be suppressed to $<400$ copies $/ \mathrm{ml}[60 \%(21 / 35)$ vs. $86 \%(30 / 35), p=0.030]$.

\section{Discussion}

This pilot trial represents the first randomized study of early vs. delayed initiation of ART in patients coinfected with HIV and TB. No cases of immune reconstitution syndrome were seen in THIRST subjects in either arm, despite diligent monitoring throughout the study period. Virologic suppression and immunologic improvements on ABC/3TC/ZDV were robust, consistent with the clear mortality benefits for 


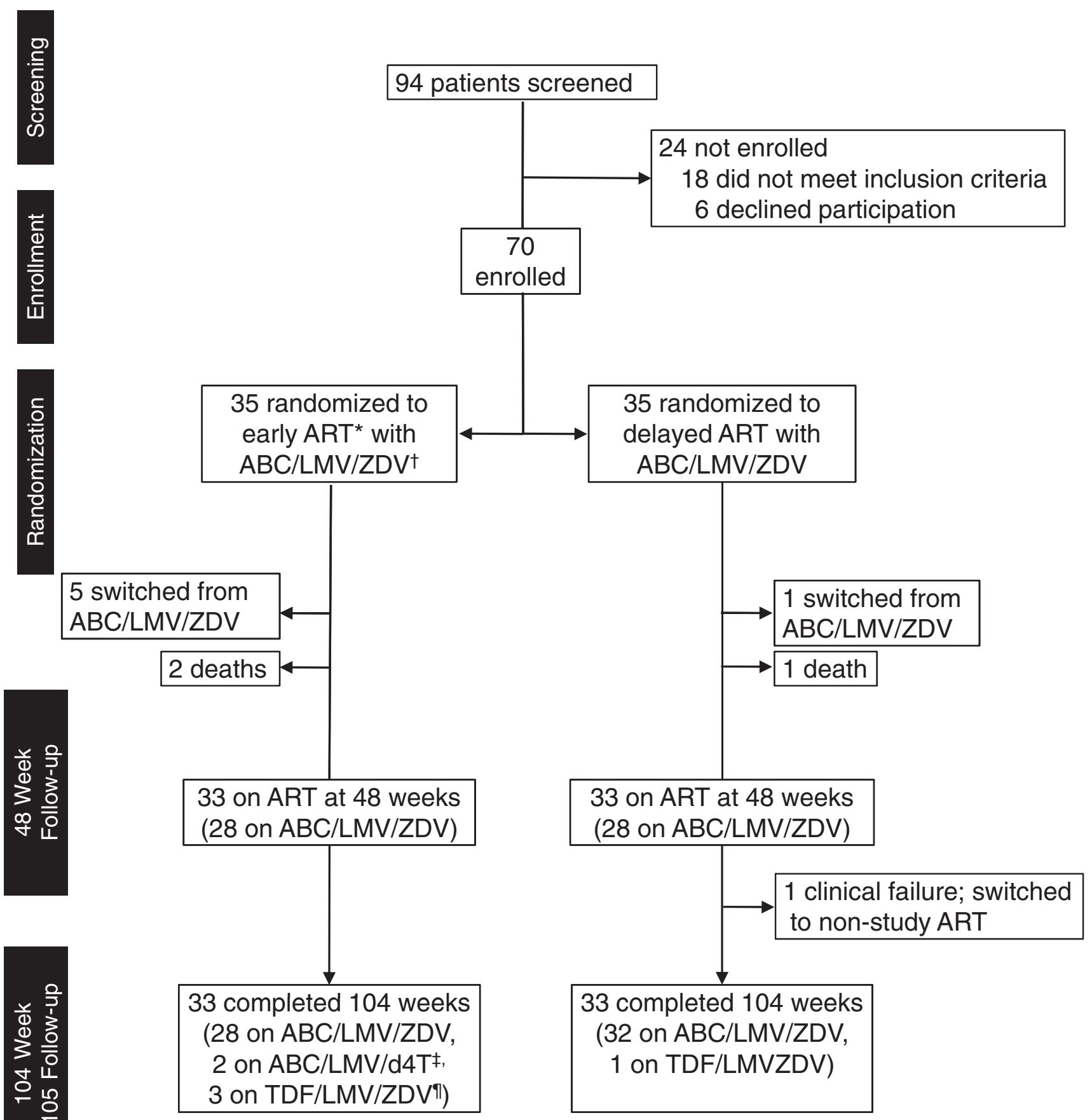

FIG. 1. Tuberculosis and HIV Immune Reconstitution Syndrome Trial (THIRST) profile and disposition of study subjects, postrandomization. ${ }^{*} \mathrm{ART}$, antiretroviral therapy; ${ }^{\dagger} \mathrm{ABC} / \mathrm{LMV} / \mathrm{ZDV}$, fixed dose combination of abacavir, lamivudine, and zidovudine; ${ }^{\star} \mathrm{ABC} / \mathrm{LMV} / \mathrm{d} 4 \mathrm{~T}$, fixed dose combination of abacavir, lamivudine, and stavudine; "TDF/LMV/ZDV, fixed dose combination of tenofovir, lamivudine, and zidovudine.

initiation of antiretrovirals in HIV and TB-coinfected populations identified in cohort studies. ${ }^{34-37}$

Concerns about the occurrence of TB-IRIS have led to confusion regarding the optimal time to start ART following initiation of anti-TB therapy. Previous cohort studies have identified these syndromes in up to $43 \%$ of coinfected persons beginning ART. ${ }^{7-14}$ THIRST sought to actively identify manifestations of immune reconstitution syndromes by keeping subjects in-hospital for the first 8 weeks of ART and included daily monitoring of symptoms and vital signs and weekly physical examinations. Therefore it is unlikely that clinically important immune reconstitution syndromes were missed among THIRST subjects. The absence of subjects with these syndromes is consistent with a more recent retrospective series $^{14}$ and two prospective trials ${ }^{32,38}$ suggesting that IRIS events occur less frequently than previously described. In 
Table 1. Baseline Demographic and Disease Characteristics at Entry of Subjects Enrolled in THIRST ${ }^{\mathrm{a}}$

\begin{tabular}{|c|c|c|c|}
\hline & $\begin{array}{l}\text { Overall } \\
(\mathrm{n}=70)\end{array}$ & $\begin{array}{l}\text { Early antiretroviral } \\
\text { therapy }(\mathrm{n}=35)\end{array}$ & $\begin{array}{c}\text { Delayed antiretroviral } \\
\text { therapy }(\mathrm{n}=35)\end{array}$ \\
\hline Male & $41(59 \%)$ & $23(66 \%)$ & $18(51 \%)$ \\
\hline Age (years) & $36.2(32.4-43.8)$ & $36.0(32.4-43.6)$ & $36.7(32.4-44.3)$ \\
\hline $\mathrm{CD} 4^{+}$cell count $\left(\right.$cells $\left./ \mathrm{mm}^{3}\right)$ & $103(55-155)$ & $106(58-151)$ & $102(51-203)$ \\
\hline Weight $(\mathrm{kg})$ & $55.5(50-61.2)$ & $54(46-60)$ & $58(51-64)$ \\
\hline Hemoglobin (g/liter) & $113(50-61.2)$ & $112(105-120)$ & $116(108-121)$ \\
\hline Erythrocyte sedimentation rate $(\mathrm{mm} / \mathrm{h})$ & $60.5(43-78.2)$ & $63(50-72)$ & $58(42-84)$ \\
\hline White blood cell count (cells $\left./ \mathrm{mm}^{3}\right)$ & $5.4(4.1-7.3)$ & $5.2(3.9-8.8)$ & $5.6(4.1-7.2)$ \\
\hline Total lymphocyte count (cells $/ \mathrm{mm}^{3}$ ) & $1.2(0.9-2.1)$ & $1.5(0.9-2.1)$ & $1.1(0.9-2.1)$ \\
\hline Creatinine $(\mu \mathrm{mol} /$ liter $)$ & $94.5(88-100)$ & $92(88-100)$ & $96(90-98)$ \\
\hline Glucose (mmol/liter) & $5.1(4.4-5.6)$ & $5.1(4.6-5.7)$ & $5.0(4.1-5.4)$ \\
\hline Alanine aminotransferase (U/liter) & $15(14-16)$ & $15(14-15.2)$ & $15(14-16)$ \\
\hline
\end{tabular}

${ }^{a}$ Data are presented as median (interquartile range), unless otherwise stated.

ACTG protocol A5164, among 282 subjects not receiving ART and presenting with acute opportunistic infection other than TB, $20(7 \%)$ of the subjects developed IRIS, ${ }^{38}$ and none of a recently described cohort of 34 subjects (with CD4 cell counts $>350 / \mathrm{mm}^{3}$ ) initiating ABC/3TC/ZDV early in their course of anti-TB therapy developed TB-IRIS. ${ }^{32}$

Three subjects died during the trial; two of them were assigned to the early ART group and had respiratory failure attributed to preexisting pulmonary disease. A third subject assigned to the delayed group died from cerebral malaria. Although it is possible that these deaths could have been directly related to or exacerbated by immune reconstitution syndromes, it is unlikely because of clearly identifiable alter-

Table 2. Serious Adverse Events Occurring Postrandomization AMONG THIRST PARTICIPANTS

\begin{tabular}{lr}
\hline Condition (N) & $\begin{array}{r}\text { Week of } \\
\text { antretroviral } \\
\text { therapy }\end{array}$ \\
\hline Early & \\
Anemia (2) & $6^{\mathrm{a}}, 13^{\mathrm{a}}$ \\
Suspected abacavir & $3^{\mathrm{a}}, 3^{\mathrm{a}}, 11^{\mathrm{a}}$ \\
$\quad$ hypersensitivity reaction (3) & 14 \\
Acute intestinal obstruction (1) & 30 \\
Amoebiasis (1) & 7 \\
Cryptococcal meningitis (1) & $12,35^{\mathrm{b}}$ \\
Malaria (2) & $20^{\mathrm{b}}$ \\
Tension pneumothorax (1) & 12 \\
Syphilis (1) & \\
Delayed & $-2^{\mathrm{c}}$ \\
Allergic reaction to & \\
$\quad$ sulfalene-pyrimethamine (1) & 86 \\
Mycobacteremia with M. sherrisii (1) & 9 \\
Esophageal variceal bleeding (1) & 5 \\
Head trauma, grand mal seizure (1) & $4^{\mathrm{b}}$ \\
Disseminated Kaposi's sarcoma (1) & $-2^{\mathrm{c}}$ \\
Malaria (1) & $2^{\mathrm{a}}$ \\
Suspected abacavir & \\
$\quad$ hypersensitivity reaction (1) & \\
\hline
\end{tabular}

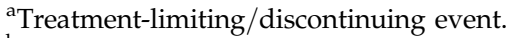

b Associated with death.

${ }^{c}$ Events occurred postrandomization, but prior to initiation of antiretroviral therapy.
}

native disease processes. Early mortality is a recognized complication of ART initiation in African cohorts, and the mortality rate of $4.3 \%$ among THIRST subjects is considerably lower than the rates of $8-26 \%$ in the first 12 months reported across multiple cohorts. ${ }^{39}$ It is also possible that the four subjects with suspected abacavir HSR could have been suffering from immune reconstitution syndromes and were mistakenly diagnosed with HSR. This is unlikely because they continued on ART with the substitution of tenofovir for abacavir, and their symptoms resolved rapidly during ongoing ART.

The antiretroviral and immunologic effects of the triple nucleoside regimen used in THIRST subjects were consistent with responses seen in other African cohorts. ${ }^{32,40}$ In the DART study, ${ }^{40}$ greater than $60 \%$ of subjects had plasma HIV RNA levels $<50$ copies $/ \mathrm{ml}$ at 48 weeks; in THIRST this trend was sustained through 104 weeks with $70 \%$ at $<50$ copies $/ \mathrm{ml}$ using a standard ITT, missing = failure, analysis. Immunologic improvement as measured by CD4 cell increases was comparable to that reported with zidovudine, lamivudine, and tenofovir in the DART trial, with a median increase of 140 cells $/ \mathrm{mm}^{3}$ at week 48 (vs. 128 cells $/ \mathrm{mm}^{3}$ reported in the DART trial). Overall, early CD4 cell increases were not as robust as has been reported in other trials in resource-limited settings. ART regimens containing zidovudine compared to those without zidovudine have been associated with smaller CD4 cell increases but not with more clinical events. ${ }^{41}$ It is possible that CD4 cell increases among THIRST subjects, all of whom were initiated on a zidovudine-containing regimen, occurred more slowly compared to patients receiving other regimens in similar settings and that a more gradual immunologic recovery favors fewer TB-IRIS events. Alternatively, patients in this study may have had smaller CD4 cell increases because of their coinfection with TB.

The risk of immune reconstitution inflammatory syndromes is greater among patients with lower baseline CD4 cell counts, 7,10,14 and the median baseline count in the THIRST subjects was 104 cells $/ \mathrm{mm}^{3}$. Therefore it is possible that immune reconstitution syndromes were not seen because of the relatively high baseline CD4 cell counts in the THIRST population. Finally, higher risk has been associated with a shorter duration of antituberculosis treatment. To be eligible for THIRST, subjects could not have received more than 14 days of anti-TB therapy; this seems like an unlikely explanation for the lack of immune reconstitution syndromes. 


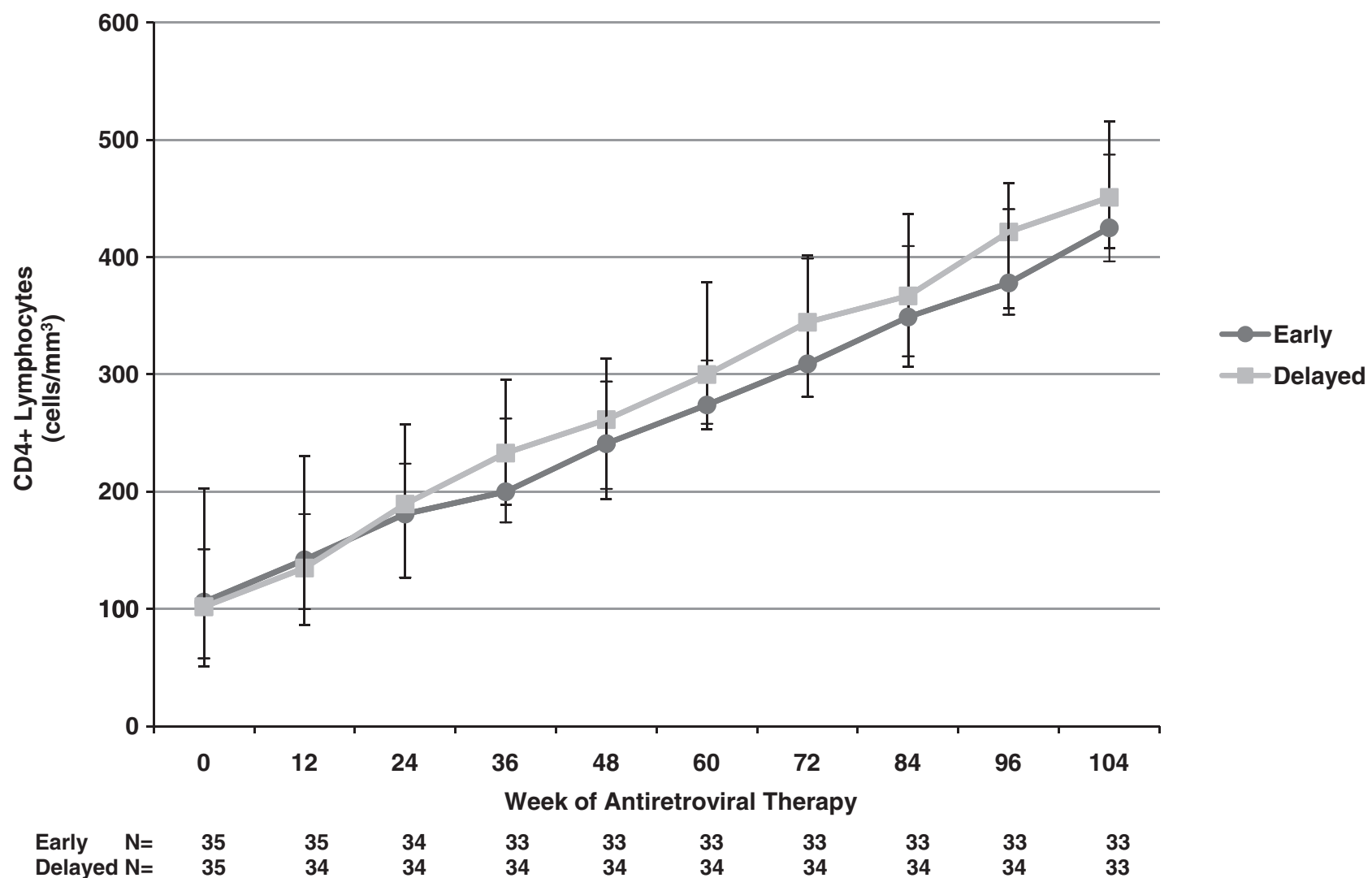

FIG. 2. Median CD4 cell counts with interquartile ranges among THIRST subjects randomized to receive early vs. delayed antiretroviral therapy in relation to initiation of antituberculosis therapy, beginning at ART initiation through 104 weeks.

Although triple nucleoside regimens have been shown to lack equivalent potency in comparison to three-drug regimens containing NNRTIs or PIs, ${ }^{15,42,43}$ they do have attributes that support their use in HIV/TB-coinfected persons. Their use is not complicated by potential drug interactions with rifampicin, and the fixed dose combination of $\mathrm{ABC} / 3 \mathrm{TC} /$ ZDV can be prescribed as a simple regimen of one pill dosed twice daily. Potential disadvantages of this regimen in coinfected persons include the occurrence of the HSR syndrome, which may be difficult to differentiate clinically from immune reconstitution disease.

The virologic and immunologic responses to $\mathrm{ABC} / 3 \mathrm{TC} /$ ZDV were consistent with previous trials of triple nucleosidecontaining and double nucleoside plus nucleotide-containing regimens. ${ }^{15,42,43}$ There were no differences in immunologic or virologic responses between the early and delayed treatment strategies. In a separate analysis in which switches from $\mathrm{ABC} / 3 \mathrm{TC} / \mathrm{ZDV}$ were considered failures, significantly more subjects randomized to early therapy demonstrated plasma HIV RNA levels $\geq 400$ copies $/ \mathrm{ml}$ at 104 weeks, suggesting that without access to appropriate substitution antiretrovirals (e.g., stavudine and tenofovir), a strategy of early ABC/3TC/ ZDV for HIV/TB-coinfected patients may be associated with greater rates of virologic failure. The regimen was well tolerated overall, with $86 \%(60 / 70)$ of subjects completing 104 weeks on $\mathrm{ABC} / 3 \mathrm{TC} / \mathrm{ZDV}$.

Four subjects $(6 \%)$ were diagnosed with suspected abacavir HSR and had abacavir switched to tenofovir; these subjects had plasma HIV RNA levels and CD4 cell counts that were similar to the subjects continuing on the triple nucleoside regimen. The suspected HSR diagnoses were made in three subjects randomized to early ART and one subject randomized to delayed ART; with such small numbers of subjects suffering HSR, no conclusions can be drawn concerning differences between the two groups. Abacavir HSR appears to be uncommon in African populations due to the low prevalence of the HLA B-5701 phenotype, ${ }^{44}$ and therefore the rate in the THIRST subjects may be unexpectedly high. Presumably this is a reflection of potentially confounding illnesses among this population coupled with the conservative management of abacavir by the study investigators.

There was a trend toward more serious adverse events in the early ART group vs. the delayed group (12 events vs. 7 events). This trend may reflect the risk of earlier initiation of ART in coinfected persons. Both subjects with zidovudineassociated anemia had increases in hemoglobin concentration following a switch from zidovudine to stavudine, and at 104 weeks they had similar responses to the subjects continuing on their original medications.

There are limitations to the THIRST study. First, the sample size is relatively small as the trial was powered to detect differences in TB-IRIS events between the two arms based on early retrospective reports of TB-IRS incidence. It was underpowered to detect small differences in immune reconstitution syndromes between the two groups, and therefore a type II error is possible. It is possible that immune reconstitution 


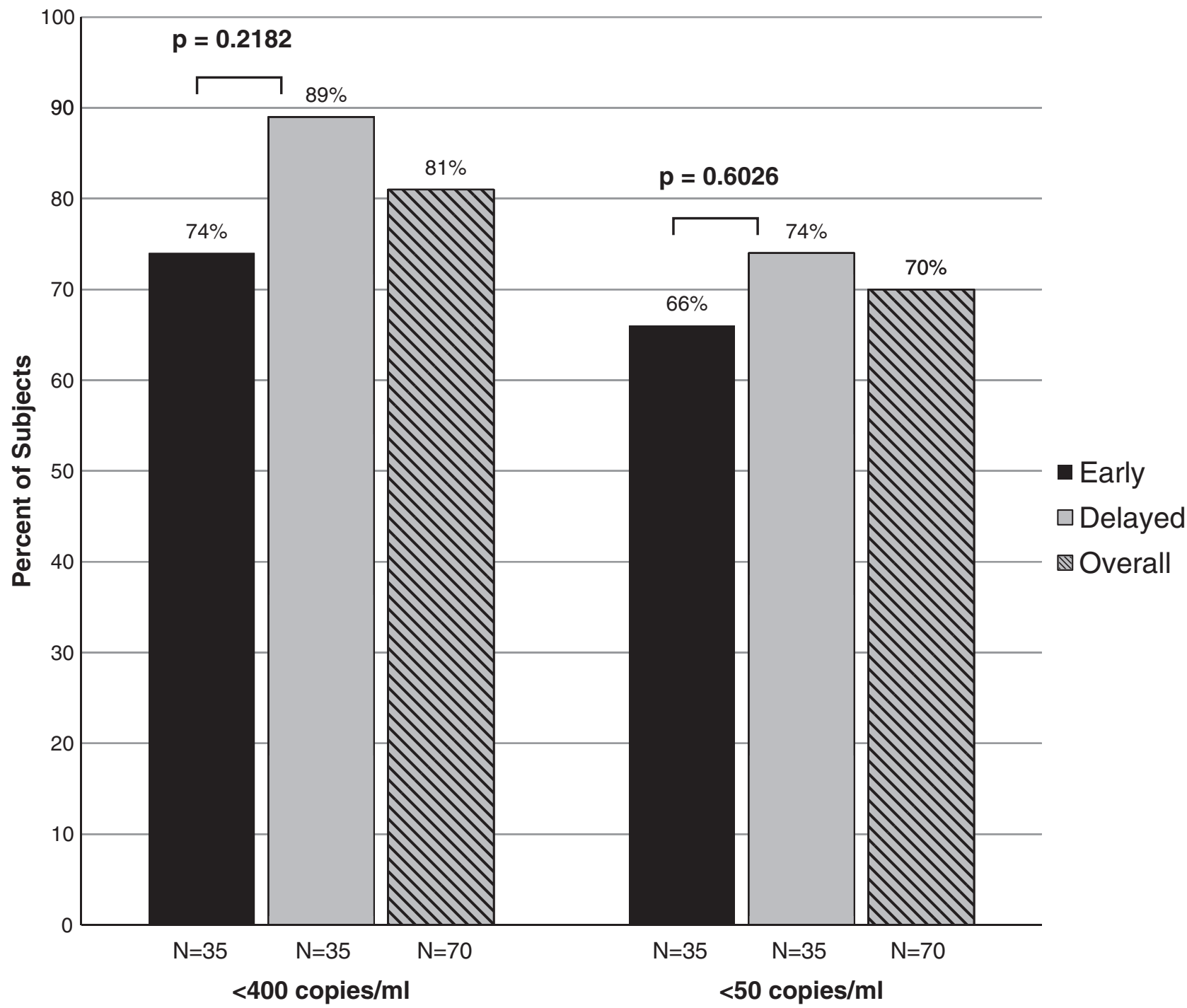

FIG. 3. Proportion of THIRST participants with plasma HIV RNA levels $<400$ copies $/ \mathrm{ml}$ and $<50$ copies/ml at 104 weeks of ART.

syndromes were missed or misdiagnosed, although the diligent surveillance in a hospitalized setting, the continuous administration of ART with tenofovir and substitution for abacavir if HSR was diagnosed, and the complete and prolonged follow-up make these possibilities unlikely. It is also possible that the exclusion criteria excluded patients most likely to develop TB-IRIS (49\% of subjects had CD4 cells counts $<100$ cells $/ \mathrm{mm}^{3}$ ). Finally, sputum culture with definite identification of Mycobacterium tuberculosis was not performed, reflecting the current standard of diagnosis in most resource-limited settings.

In summary, the THIRST results suggest that early ART can be well tolerated by HIV/TB-coinfected subjects with a low risk of immune reconstitution syndromes, although there may be more adverse events necessitating regimen switches with early ART. Early initiation of ART led to predictable benefits in virologic suppression and increases in CD4 cell counts among THIRST study subjects receiving the triple nucleoside combination of $\mathrm{ABC} / 3 \mathrm{TC} / \mathrm{ZDV}$ with appropriate substitutions for drug toxicity. Larger, fully powered studies are needed to provide a definitive answer to the clinical question of when to start ART, but the THIRST results provide additional evidence to support the initiation of ART without delay.

\section{Acknowledgments}

This research was supported by GlaxoSmithKline. Investigators received additional support from the following United States National Institutes of Health (NIH) programs: International Studies on AIDS Associated Co-infections (ISAAC) award (U01 AI062563; J.A.B., J.A.C., H.R., H.S., J.S., and N.M.T.); AIDS International Training and Research Program grant ( D43 PA-03-018; J.A.B., J.A.C., H.R., H.S., J.S., and N.M.T.); the Duke AIDS Clinical Trials Unit and Clinical Research Sites (U01 AI069484-01; J.A.B., J.A.C., and N.M.T.); and the Duke Center for AIDS Research (CFAR) award (P30 AI064518; J.A.B.). Presented in part at the 13th Conference on Retroviruses and Opportunistic Infections (paper \#796), Denver, February 5-8, 2006. 


\section{Disclosure Statement}

Duke University, Durham, NC, received funding from GlaxoSmithKline to support the THIRST study, which was investigator-initiated (J.A.B and N.M.T). H.S. has received speaking honorarium from GlaxoSmithKline. J.B. has received research support, consulting fees, and speaking honoraria from Abbott Laboratories and consulting fees from Pfizer. N.M.T. has received consulting fees and speaking honoraria from Gilead and Abbott. We are not aware of any other potential conflicts.

\section{References}

1. World Health Organization. Global Tuberculosis Control: Surveillance, Planning, Financing, 2008. http://www.who. int/tb/publications/global_report/en/. Accessed August 18, 2009.

2. Ansari NA, Kombe AH, Kenyon TA, et al:: Pathology and causes of death in a group of 128 predominantly HIVpositive patients in Botswana, 1997-1998. Int J Tuberc Lung Dis 2002;6:55-63.

3. Corbett EL, Marston B, Churchyard GJ, and De Cock KM: Tuberculosis in sub-Saharan Africa: Opportunities, challenges, and change in the era of antiretroviral treatment. Lancet 2006;367:926-937.

4. Grant AD, Djomand G, and De Cock KM: Natural history and spectrum of disease in adults with HIV/AIDS in Africa. AIDS 1997;11(Suppl B):S43-S54.

5. Ole-Nguyaine S, Crump JA, Kibiki GS, et al.: HIV-associated morbidity, mortality, and diagnostic testing opportunities among inpatients at a referral hospital in northern Tanzania. Ann Trop Med Parasitol 2004;98:171-179.

6. Rana FS, Hawken MP, Mwachari C, et al.: Autopsy study of HIV-1-positive and HIV-1-negative adult medical patients in Nairobi, Kenya. J Acquir Immune Defic Syndr 2000;24:23-29.

7. Breton G, Duval X, Estellat C, et al.: Determinants of immune reconstitution inflammatory syndrome in HIV type 1-infected patients with tuberculosis after initiation of antiretroviral therapy. Clin Infect Dis 2004;39:1709-1712.

8. Kumarasamy $\mathrm{N}$, Chaguturu S, Mayer $\mathrm{KH}$, et al:: Incidence of immune reconstitution syndrome in HIV/tuberculosiscoinfected patients after initiation of generic antiretroviral therapy in India. J Acquir Immune Defic Syndr 2004;37:1574-1576.

9. Mcllleron H, Meintjes G, Burman WJ, and Maartens G: Complications of antiretroviral therapy in patients with tuberculosis: Drug interactions, toxicity, and immune reconstitution inflammatory syndrome. J Infect Dis 2007; 196(Suppl 1):S63-S75.

10. Michailidis C, Pozniak AL, Mandalia S, Basnayake S, Nelson MR, and Gazzard BG: Clinical characteristics of IRIS syndrome in patients with HIV and tuberculosis. Antivir Ther 2005;10:417-422.

11. Narita M, Ashkin D, Hollender ES, and Pitchenik AE: Paradoxical worsening of tuberculosis following antiretroviral therapy in patients with AIDS. Am J Respir Crit Care Med 1998;158:157-161.

12. Navas E, Martin-Davila P, Moreno L, et al.: Paradoxical reactions of tuberculosis in patients with the acquired immunodeficiency syndrome who are treated with highly active antiretroviral therapy. Arch Intern Med 2002;162:97-99.

13. Olalla J, Pulido F, Rubio R, et al.: Paradoxical responses in a cohort of HIV-1-infected patients with mycobacterial disease. Int J Tuberc Lung Dis 2002;6:71-75.
14. Lawn SD, Myer L, Bekker LG, and Wood R: Tuberculosisassociated immune reconstitution disease: Incidence, risk factors and impact in an antiretroviral treatment service in South Africa. AIDS 2007;21:335-341.

15. Gulick RM, Mellors JW, Havlir D, et al.: Treatment with indinavir, zidovudine, and lamivudine in adults with human immunodeficiency virus infection and prior antiretroviral therapy. N Engl J Med 1997;337:734-739.

16. Hammer SM, Squires KE, Hughes MD, et al.: A controlled trial of two nucleoside analogues plus indinavir in persons with human immunodeficiency virus infection and CD4 cell counts of 200 per cubic millimeter or less. AIDS Clinical Trials Group 320 Study Team. N Engl J Med 1997;337:725733.

17. Mocroft A, Vella S, Benfield TL, et al.: Changing patterns of mortality across Europe in patients infected with HIV-1. EuroSIDA Study Group. Lancet 1998;352:1725-1730.

18. Braitstein P, Brinkhof MW, Dabis F, et al.: Mortality of HIV1-infected patients in the first year of antiretroviral therapy: Comparison between low-income and high-income countries. Lancet 2006;367:817-824.

19. Breen RA, Smith CJ, Bettinson H, et al:: Paradoxical reactions during tuberculosis treatment in patients with and without HIV co-infection. Thorax 2004;59:704-707.

20. Shelburne SA, Visnegarwala F, Darcourt J, et al.: Incidence and risk factors for immune reconstitution inflammatory syndrome during highly active antiretroviral therapy. AIDS 2005;19:399-406.

21. Ackah AN, Coulibaly D, Digbeu $\mathrm{H}$, et al.: Response to treatment, mortality, and CD4 lymphocyte counts in HIVinfected persons with tuberculosis in Abidjan, Cote d'Ivoire. Lancet 1995;345:607-610.

22. Perriens JH, St Louis ME, Mukadi YB, et al:: Pulmonary tuberculosis in HIV infected patients in Zaire. A controlled trial of treatment for either 6 or 12 months. N Engl J Med 1995;332:779-784.

23. Chaisson RE, Clermont HC, Holt EA, et al.: Six-month supervised intermittent tuberculosis therapy in Haitian patients with and without HIV infection. Am J Respir Crit Care Med 1996;154:1034-1038.

24. Centers for Disease Control and Prevention: Managing Drug Interactions in the Treatment of HIV-Related Tuberculosis, 2007. http://www.cdc.gov/tb/publications/guidelines/TB_ HIV_Drugs/PDF/tbhiv.pdf. Accessed August 18, 2009.

25. Harries AD, Maher D, and Graham S: TB/HIV: A Clinical Manual, 2nd ed. World Health Organization, 2004.

26. World Health Organization: Scaling Up Antiretroviral Therapy in Resource-Limited Settings: Treatment Guidelines for a Public Health Approach (2003 Revision), 2004. http://www. who.int/3by5/publications/documents/arv_guidelines/en/ index.html. Accessed August 18, 2009.

27. Bristol-Myers Squibb: Sustiva [package insert], 2008.

28. Gupta R, Hill A, Sawyer AW, and Pillay D: Emergence of drug resistance in HIV type 1-infected patients after receipt of first-line highly active antiretroviral therapy: A systematic review of clinical trials. Clin Infect Dis 2008;47:712-722.

29. Boulle A, Orrel C, Kaplan R, et al.: Substitutions due to antiretroviral toxicity or contraindication in the first 3 years of antiretroviral therapy in a large South African cohort. Antivir Ther 2007;12:753-760.

30. World Health Organization: Transaction Prices for Antiretroviral Medicines and HIV Diagnostics from 2004 to September 2008. http://www.who.int/hiv/amds/GPRM summaryReportOct2008.pdf. Accessed August 18, 2009. 
31. Renaud-Thery F, Nguimfack BD, Vitoria M, et al.: Use of antiretroviral therapy in resource-limited countries in 2006: Distribution and uptake of first- and second-line regimens. AIDS 2007;21(Suppl 4):S89-S95.

32. Srikantiah P, Walusimbi MN, Kayanja HK, et al.: Early virological response of zidovudine/lamivudine/abacavir for patients co-infected with HIV and tuberculosis in Uganda. AIDS 2007;21:1972-1974.

33. Crump J, van Ingen J, Morrissey A, et al.: Invasive disease caused by nontuberculous mycobacteria in HIV-infected patients in Tanzania. Emerg Infect Dis 2009;15:53-55.

34. Dean GL, Edwards SG, Ives NJ, et al.: Treatment of tuberculosis in HIV-infected persons in the era of highly active antiretroviral therapy. AIDS 2002;16:75-83.

35. Dheda K, Lampe FC, Johnson MA, and Lipman MC: Outcome of HIV-associated tuberculosis in the era of highly active antiretroviral therapy. J Infect Dis 2004;190:1670-1676.

36. Akksilp S, Karnkawinpong O, Wattanaamornkiat W, et al.: Antiretroviral therapy during tuberculosis treatment and marked reduction in death rate of HIV-infected patients, Thailand. Emerg Infect Dis 2007;13:1001-1007.

37. Sanguanwongse N, Cain KP, Suriya P, et al.: Antiretroviral therapy for HIV-infected tuberculosis patients saves lives but needs to be used more frequently in Thailand. J Acquir Immune Defic Syndr 2008;48:181-189.

38. Zolopa A, Andersen J, Komarow L, et al.: Early antiretroviral therapy reduces AIDS progression/death in individuals with acute opportunistic infections: A multicenter randomized strategy. PLOS ONE 2009;4:e5575.

39. Lawn SD, Harries AD, Anglaret $X$, Myer L, and Wood R: Early mortality among adults accessing antiretroviral treat- ment programmes in sub-Saharan Africa. AIDS 2008;22: 1897-1908.

40. DART Virology Group and Trial Team: Virological response to a triple nucleoside/nucleotide analogue regimen over 48 weeks in HIV-1-infected adults in Africa. AIDS 2006;20: 1391-1399.

41. Huttner AC, Kaufmann GR, Battegay M, Weber R, and Opravil M: Treatment initiation with zidovudine-containing potent antiretroviral therapy impairs CD4 cell count recovery but not clinical efficacy. AIDS 2007;21:939-946.

42. Staszewski S, Keiser P, Montaner J, et al.: Abacavirlamivudine-zidovudine vs indinavir-lamivudine-zidovudine in antiretroviral-naive HIV-infected adults: A randomized equivalence trial. JAMA 2001;285:1155-1163.

43. Bartlett JA, Johnson J, Herrera G, et al.: Long-term results of initial therapy with abacavir and lamivudine combined with efavirenz, amprenavir/ritonavir, or stavudine. J Acquir Immune Defic Syndr 2006;43:284-292.

44. Young B, Squires K, Patel P, et al.: First large, multicenter, open-label study utilizing HLA-B $* 5701$ screening for abacavir hypersensitivity in North America. AIDS 2008;22:1673-1675.

Address correspondence to: Nathan M. Thielman Department of Medicine

Division of Infectious Diseases and International Health Box 90519, Room 109, Trent Hall Duke University Medical Center Durham, North Carolina 27708

E-mail: n.thielman@duke.edu 
